\title{
Aesthetic Correction of Mild-to-Moderate Blepharoptosis Among Asians: The Bridge Technique
}

\author{
Yirui Shen $\cdot$ Wenjie Yu $\cdot$ Feixue Ding $\cdot$ Lin Lu $\cdot$ Fei Liu \\ Di Sun $\cdot$ Xusong Luo $\cdot$ Rui Jin $\cdot$ Jun Yang
}

Received: August 19, 2021 / Accepted: October 21, 2021 / Published online: November 6, 2021

(C) The Author(s) 2021

\begin{abstract}
Introduction: Although several surgical methods have been introduced to treat mild-tomoderate blepharoptosis, including levatorbased techniques such as Müller muscle-conjunctival resection, few complications and better functional and aesthetic outcomes remain elusive for plastic surgeons. Hence, this study aimed to provide a new technique (bridge technique) using the levator aponeurosis-Müller's muscle flap to achieve optimal blepharoptosis correction for function and aesthetics among Asians.
\end{abstract}

Yirui Shen and Wenjie Yu contributed equally to this work.

Supplementary Information The online version contains supplementary material available at https:// doi.org/10.1007/s40123-021-00417-3.

Y. Shen · F. Ding - L. Lu - F. Liu - D. Sun - X. Luo · R. Jin $(\varangle) \cdot$ J. Yang $(\bowtie)$

Department of Plastic and Reconstructive Surgery, Shanghai Ninth People's Hospital Affiliated to Shanghai Jiaotong University School of Medicine, 639 Zhizaoju Road, Shanghai 200011, China e-mail: dr.jinrui@hotmail.com

J. Yang

e-mail: yj55569@hotmail.com

W. Yu

Mylike Medical Cosmetic Hospital, Shanghai, China
Methods: From January 2019 to May 2020, this new technique was performed on 157 consecutive patients with mild-to-moderate blepharoptosis. Our technique was based on the anchor of the levator complex to the tarsus using mattress stitches and three-layer fixation, which provided a reliable motion transmitter for elevating the upper eyelid. The patients' medical records and photographs were reviewed 12 months postoperatively to assess the margin reflex distance 1 (MRD1), incidence of complications, and aesthetic outcomes.

Results: The average preoperative and postoperative MRD1 measured $1.2 \pm 0.31 \mathrm{~mm}$ and $3.7 \pm 0.34 \mathrm{~mm}$, respectively. A significant difference was observed between the preoperative and postoperative distance values $(p<0.001)$. In the aesthetic evaluation, the grading was good, fair, and poor for $142(90.4 \%)$ patients, 10 (6.4\%) patients, and 5 (3.2\%) patients, respectively. Complications included undercorrection in $12(5.2 \%)$ cases and overcorrection in nine (3.9\%) cases, and no cases of residual lagophthalmos were recorded.

Conclusion: Mild-to-moderate blepharoptosis among Asians can be corrected effectively using this new technique. However, the long-term outcomes of this procedure should be explored.

Keywords: Blepharoptosis; Bridge technique; Levator complex; Oculoplastic 


\section{Key Summary Points}

Why carry out this study?

Although mild-to-moderate blepharoptosis can be corrected by several methods, complications such as undercorrection and recurrence remain.

Traditional levator-based techniques can result in asymmetric scars and deep-set creases in Asians owing to the fullness of the upper eyelid.

\section{What was learned from the study?}

This study presents a new surgical technique that features an aponeurotic flap linkage that requires the levator aponeurosis and Müller's muscle to be reanchored to both the tarsus and skin by enhanced three-layer fixation.

This new technique is effective in correcting mild-to-moderate blepharoptosis and can achieve optimal aesthetic outcomes among Asians.

\section{DIGITAL FEATURES}

This article is published with digital features, including a video, to facilitate understanding of the article. To view digital features for this article, go to https://doi.org/10.6084/m9. figshare.16847263.

\section{INTRODUCTION}

Blepharoptosis is defined by either unilateral or bilateral drooping of the upper eyelid, which can lead to aesthetic discomfort or even functional visual deficits [1]. Generally, Müllerectomy and levator-based techniques are applied for mild-to-moderate blepharoptosis with fairto-good levator function [2].

Although most blepharoptosis cases can be effectively corrected using these methods,

complications such as undercorrection and recurrence remain [3]. The reoperation rates of levator-based techniques reportedly varied from 3 to $18 \%$ [4-7].

Moreover, patients currently have more stringent aesthetic demands in addition to their need for functional correction in blepharoptosis surgeries, which is especially true in young Asian patients with mild-to-moderate blepharoptosis, who demand a more natural and dynamic postoperative palpebral shape [8]. Furthermore, the anatomy of the upper eyelid in Asians, which is characterised by the absence of or a very low eyelid crease and puffier upper eyelid appearance, is different from that of Caucasians. The fullness of the Asian upper eyelid is attributed to the drooping preaponeurotic fat, which is prominently subcutaneous, suborbicularis, and pretarsal fat tissue [9]. Traditional surgical procedures only focus on enhancement of the levator function, while ignoring the double eyelid shape, which could result in a deep-set and unsightly double eyelid crease on the puffier upper eyelid.

Understanding these anatomical differences between Asians and Caucasians and the deficiencies of traditional techniques is the key to achieving successful correction of blepharoptosis and creating natural double eyelids, while maintaining the ethnic characteristics of Asians. Hence, we aimed to present a new surgical technique that features an aponeurotic flap linkage that requires the levator aponeurosis and Müller's muscle to be re-anchored to both the tarsus and skin by enhanced three-layer fixation. The aponeurotic flap was terminated at the skin and clamped by the orbicularis oculi muscle, thus achieving anatomical and aesthetic correction of blepharoptosis. This technique is later referred as the "bridge technique" because the levator aponeurosis-Müller's muscle flap serves as a bridge attached to the upper eyelid retractors and the other end fixed to orbicularis oculi muscle and skin. This technique has been used in our department since January 2019 and has proven to be effective in correcting mild-to-moderate blepharoptosis with minimal scar formation. 


\section{METHODS}

From January 2019 to May 2020, 157 consecutive patients with mild-to-moderate blepharoptosis were included in this study. The inclusion criteria were as follows: (1) > 18 years of age, (2) primary surgery for eyelids, (3) levator function from 4 to $7 \mathrm{~mm}$, and (4) not suffering from myasthenia gravis, Marcus Gunn syndrome, or glaucoma. All the patients provided signed informed consent. In this study, all procedures involving human participants were conducted in accordance with the Declaration of Helsinki and were approved by the Ethics Committee of Shanghai Ninth People's Hospital Affiliated to Shanghai Jiaotong University School of Medicine.

For preoperative evaluation, detailed historytaking and ophthalmologic examinations were performed. The degree of ptosis was measured by the distance of the upper eyelid margin below the superior corneal limbus [10]. The normal position of the upper eyelid margin was $1 \mathrm{~mm}$ to $2 \mathrm{~mm}$ below the superior corneal limbus in the primary gaze position. The degree of blepharoptosis was determined according to margin reflex distance 1 (MRD1), which is measured with a ruler. It was categorised as mild $(\geq 2 \mathrm{~mm})$, moderate $(\geq 1 \mathrm{~mm})$, or severe $(<1 \mathrm{~mm})$ [11]. Levator function and ocular protection detected by Bell's phenomenon were also evaluated. Levator function was assessed by firmly securing the thumb over the brow while measuring the excursion of the upper eyelid from the maximal down gaze to the maximal up gaze position.

\section{Surgical Technique}

The incision height was designed based on whether the patient had unilateral or bilateral blepharoptosis. For patients with bilateral blepharoptosis, the incision lines were marked along the existing upper eyelid skin creases or were newly designed 5-7 $\mathrm{mm}$ above the eyelid margin if the crease was absent. For patients with unilateral blepharoptosis, the incision lines were $1 \mathrm{~mm}$ lower than the crease on the unaffected side.
Local anaesthesia was achieved by subcutaneous injection of $2 \%$ lidocaine (containing 1:100,000 epinephrine) $2 \mathrm{ml}$ each side. Along the marked line, a skin incision was made with a sharp blade. Monopolar electrical cautery was used to open and elevate the orbicularis oculi muscle. Then, the orbital septum was exposed and opened at the lowest part using cutting cautery (Fig. 1a). The orbicularis oculi muscle was then dissected inferiorly until 1-2 mm above the upper eyelid margin to expose the tarsus (Fig. 2b). Subsequently, the levator aponeurosis-Müller's muscle complex was carefully detached from the anterior surface of the tarsus to avoid cutting into the supratarsal arcade, thus preventing unnecessary bleeding. During this dissection, a corneal protector should be used for continuous dissection of the flap along the palpebral conjunctival surface without opening the conjunctiva. At this point, a flap containing both the levator aponeurosis and Müller's muscle was elevated (Fig. 1c). Further, an adjustable suture of the flap was located 1-2 mm below the superior border of the tarsus with mattress sutures using 6-0 non-absorbable braided silk. The patient was asked to open their eyes to check for double eyelid height and curvature. The height was adjusted according to the extent of intraoperative corneal exposure. The adjusted upper eyelid margin should be on the superior corneal limbus or $1 \mathrm{~mm}$ higher than that on the unaffected side. A total of five stitches by mattress sutures were applied to complete the construction and anchoring of the bridge. Specifically, the locations of the five stitches on the tarsus were as follows: (1) the central point of the pupil, (2) the inner limbus, (3) the lateral limbus, (4) the midpoint of the lateral limbus and lateral commissure, and (5) the midpoint of the inner limbus and medial commissure. After tarsal fixation, the redundant flap was trimmed to $1-2 \mathrm{~mm}$ and was then inserted into the skin muscle layer. For internal fixation, interrupted 6-0 polydioxanone sutures were applied. The needle passed through the orbicularis oculi muscle of the lower eyelid flap, the bottom of the anchored flap, and the orbicularis oculi muscle of the upper eyelid flap (Fig. 1d). In the end, the skin-top of the anchored flap-skin was stitched using a 7-0 

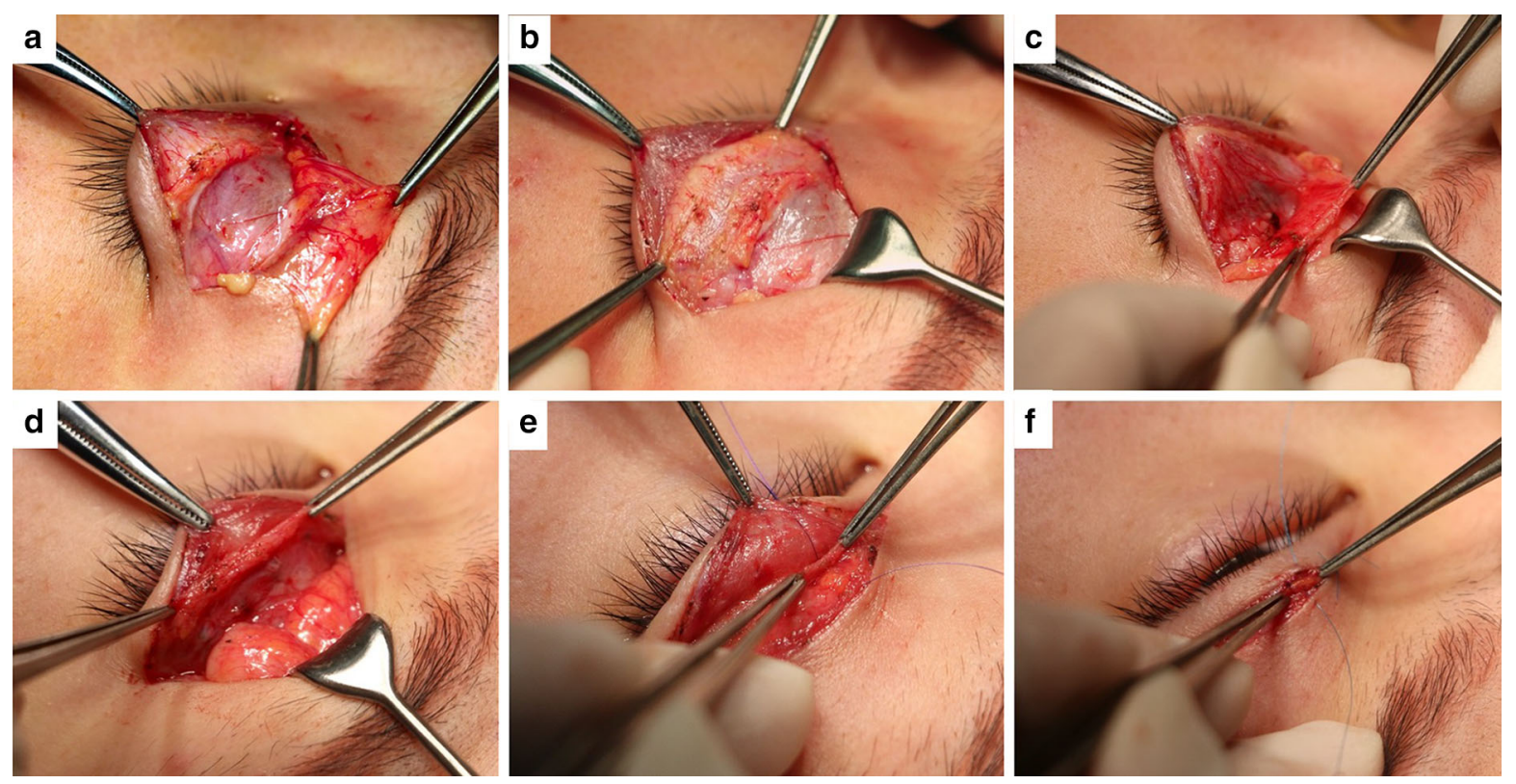

Fig. 1 The operation procedure on an 18-year-old female patient with unilateral blepharoptosis: a Opening of the orbital septum. b Exposure of the tarsus. c The formation of the levator aponeurosis-Müller's muscle flap (clamped by two forceps). $\mathbf{d}$ The flap was anchored to the tarsus by 5

Prolene to close the incision. As a result, the orbicularis oculi muscles clamped the flap (Fig. 1e). The operation was performed by a senior surgeon, and the operating time was approximately $1 \mathrm{~h}$ per eye.

A diagram of the entire operation procedure is presented in Fig. 2, and a video of the operation procedure is provided.

\section{Postoperative Care}

Immediately after the operation, erythromycin was applied to close the eyelids for $24 \mathrm{~h}$. For patients with lagophthalmos $>2 \mathrm{~mm}$ or for patients with negative or suspicious positive Bell phenomenon, the upper and lower eyelids were closed using the Frost suture [12].

After removing the gauze patch the second day, the patients needed to apply eye drops four times a day. Erythromycin ointment and Frost sutures were always applied in the evening to protect the eye from corneal exposure until the lagophthalmos regressed during sleep. stitches using mattress sutures. e Internal fixation was completed. f Additional stitches were applied to close the incision

\section{Outcome Assessments}

Outcome assessments concerned the following four aspects: (1) MRD1 was the primary outcome, (2) recurrence rate, (3) incidence of complications, and (4) secondary aesthetic outcomes. Aesthetic outcomes were graded according to the following three aspects: symmetry, eyelid contour, and scar formation. Two independent plastic surgeons (L.L. and R.J.) assessed patients at the 1-year follow-up by reviewing the photos and medical records. Photographs of the patients were taken in sitting position with primary gaze using a digital camera (M50, Canon, Japan).

Preoperative and postoperative MRD1 measurements were compared using paired $t$ tests, which were performed by the SPSS version 10.0 (SPSS, Chicago, IL, USA).

The aesthetic criteria for the outcomes are listed in Table 1.

- Good: The eyelid shape is well-formed and natural looking with appropriately sized 
a

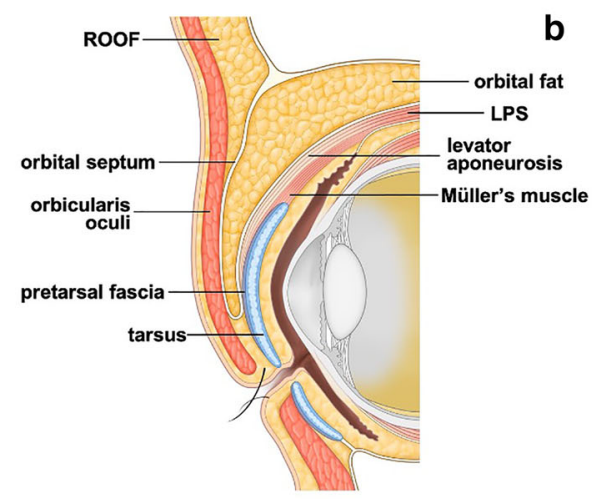

d

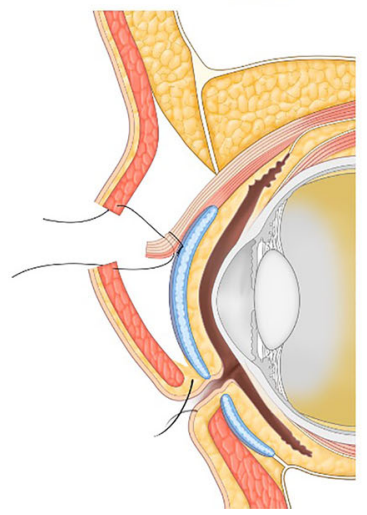

b

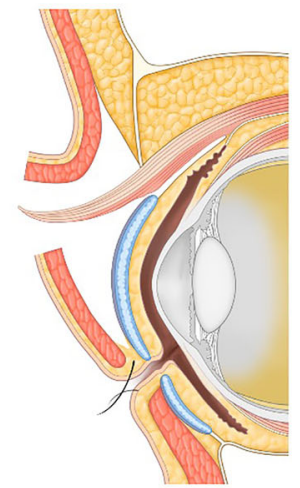

e

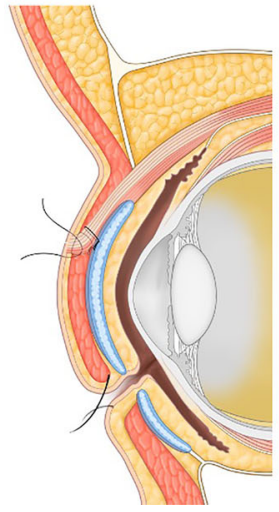

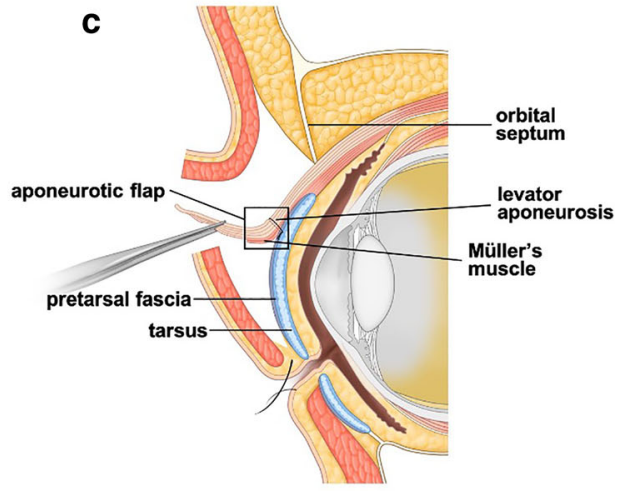

f

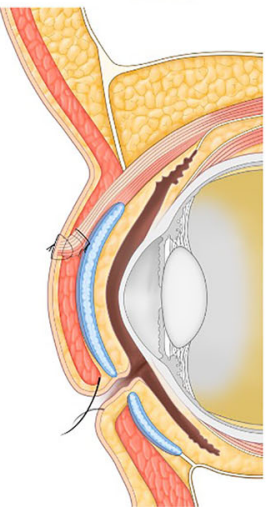

Fig. 2 Diagram of the entire operation procedure. a Upper eyelid anatomy. $\mathbf{b}$ Incision was made, and the orbicularis muscle was dissected. Levator aponeurosis-Müller's muscle complex was carefully detached from the tarsus. c Fixation of the flap to tarsus. $\mathbf{d}$ The overlong flap was trimmed, and the internal fixation was completed. e External fixation was completed through additional stitches. f Postoperative view. ROOF, retro-orbicularis oculi fat. LPS, levator palpebrae superioris. Figure credit: Min Liu

Table 1 Aesthetic grade distributions

\begin{tabular}{llll}
\hline & Good & Fair & Poor \\
\hline Eyelid contour & Well-formed and natural looking & Well-formed & Poor eyelid shape \\
Symmetry & Symmetry & Slight asymmetry & Obvious asymmetry \\
Scar formation & Flat and unnoticeable & Acceptable & Notched and uneven \\
\hline
\end{tabular}

length and width dimensions; bilateral eyelid creases are symmetrical; the incision scar is flat and unnoticeable.

- Fair: The eyelid shape is well-formed, although there is a slight asymmetry (MRD1 compared with the contralateral eyelid was between 1 and $2 \mathrm{~mm}$ ) in length or width dimensions; the incision scar is visible but acceptable.
- Poor: The eyelid shape is poor or has evident bilateral asymmetry (the difference in MRD1 was $>2 \mathrm{~mm}$ ); the incision scar was notched and uneven.

The assessed complications included undercorrection, overcorrection, exposure keratitis, residual lagophthalmos, and any combination of complications. Undercorrection refers to a result in which the patient's upper eyelid 
Table 2 Demographic characteristics of patients

\begin{tabular}{ll}
\hline No. of patients/eyes & $157 / 225$ \\
Average age (years) & $25.1(18-35)$ \\
Male/female & $51 / 106$ \\
Unilateral/bilateral & $89 / 68$ \\
Degree of blepharoptosis & \\
Mild & 34 \\
Moderate & 123 \\
\hline
\end{tabular}

margin is $>2 \mathrm{~mm}$ below the superior corneal limbus. Overcorrection refers to a postoperative result in which the upper eyelid margin lies above the superior corneal limbus. Recurrence was defined as when the patient's MRD1 returned to the preoperative level. Video followups were conducted to evaluate whether patients had keratitis or lagophthalmos at 1, 3, and 6 months following surgery. One year after surgery, the patients were asked to visit the hospital for aesthetic evaluation and MRD1 measurement.

\section{RESULTS}

Patient demographic characteristics are presented in Table 2. Altogether, 157 (225 eyes) patients were treated using our new surgical technique. Here, 89 patients had unilateral blepharoptosis, and 68 patients had bilateral blepharoptosis. The patients had a median age of 25.1 years (18-35 years) and a sex ratio of 0.48 male/female. The blepharoptosis was mild in 34 patients and moderate in 123 patients. The average follow-up period was 15.4 (12-24) months (Figs. 3, 4).

The mean preoperative MRD $1 \pm$ standard deviation (SD) measured $1.2 \pm 0.31 \mathrm{~mm}$, and the mean postoperative MRD1 was $3.7 \pm 0.34 \mathrm{~mm}(p<0.001)$.

With regard to the aesthetic evaluation, 142 (90.4\%) patients were graded as good and 10 (6.4\%) patients were graded as fair, four of whom had unnatural contour with upper eyelid deformity, and six of whom had a slight asymmetry. Five $(3.2 \%)$ patients were graded as poor, two of whom had notched scars, and the other three had evident asymmetry (Table 3).

With regard to the evaluation of complications, 12 (5.3\%) eyes featured undercorrection, 9 (4\%) eyes showed overcorrection, and 0 eyes presented with exposure keratitis. Temporary lagophthalmos was present in 10 eyes, and in most cases, lagophthalmos regressed spontaneously after 3-6 months. No cases of residual lagophthalmos were recorded at the 12-month visit, while 10 eyes showed recurrence (4.4\%) (Table 4).

\section{DISCUSSION}

This study demonstrated that our new technique is an effective method for mild-to-moderate blepharoptosis correction, considering the cosmetics and function among young Asians. However, this technique warrants careful dissection and three-layer fixation, which demands operator familiarity with upper eyelid anatomy, and could be challenging for beginners.

Several noteworthy key points for this technique are as follows:

1. The designed incision line should be $1 \mathrm{~mm}$ lower than that on the unaffected side, since the tightened skin that is attributed to enhancement of the levator function would inevitably widen the incision height.

2. Excessive injection of the anaesthetic or overswelling during the operation affected the judgement of the amount of advancement, which could cause asymmetry.

3. Electrical cautery was preferred, as it can reduce the chance of bleeding and ensure a clear operative view. The supratarsal arcade should be avoided when dissecting the flap near the superior border of the tarsus.

4. When dissecting close to the superior conjunctival fornix, injury to the superior rectus muscle should be avoided, as damage to this muscle could cause diplopia after surgery.

5. When elevating the levator aponeurosis-Müller's muscle flap, special attention should be paid to the palpebral part of the 

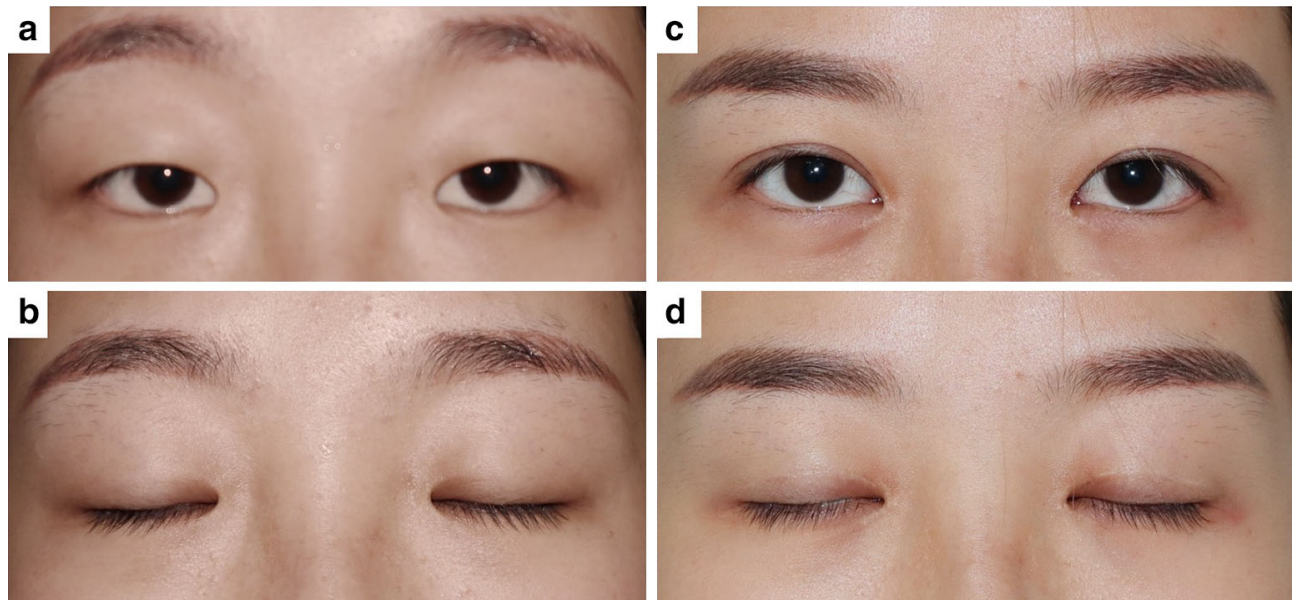

Fig. 3 A female patient (23 years old) who had moderate bilateral blepharoptosis and epicanthus. a, b Preoperative photos with her eyes open and closed. c, d Postoperative

photos with eyes open and closed 12 months after blepharoptosis surgery combined with epicanthoplasty
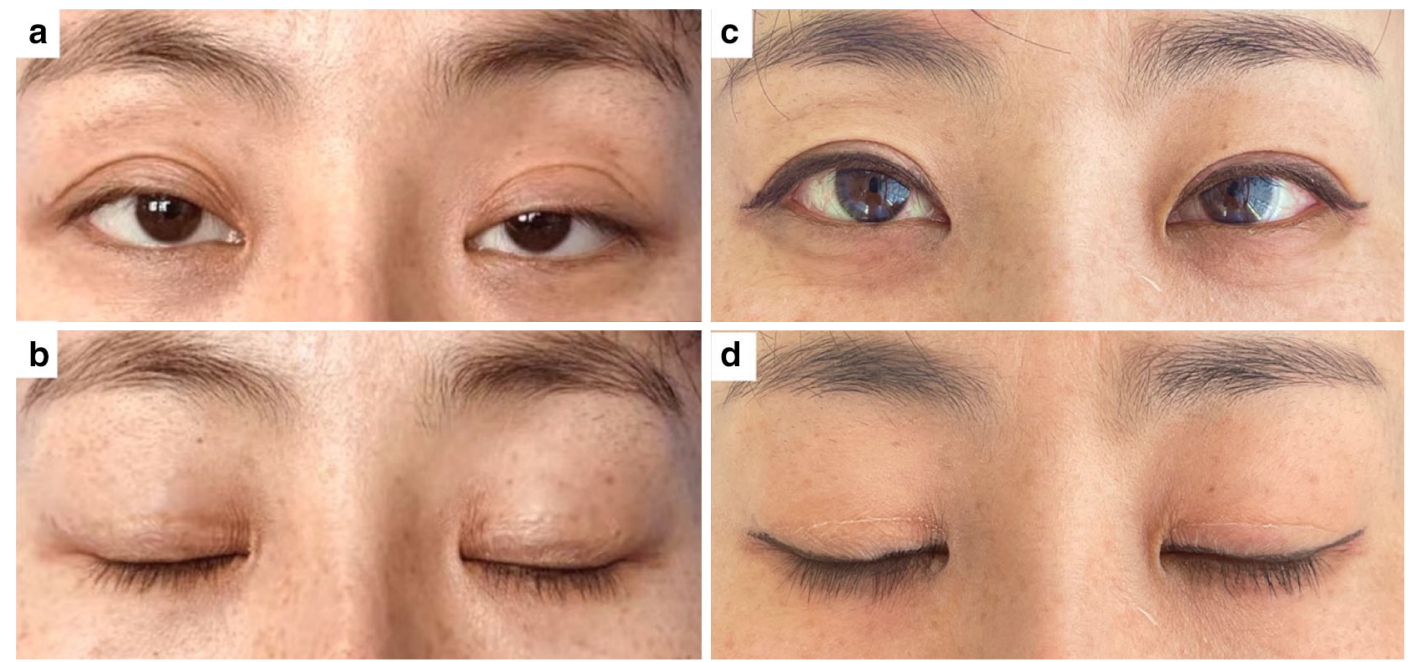

Fig. 4 A 38-year-old female patient who had unilateral blepharoptosis and positive Bell phenomenon. The left eye was graded as moderate blepharoptosis. a Preoperative

Table 3 Results of aesthetic outcomes ( $N=157$ patients)

\begin{tabular}{lll}
\hline No. of patients (rate) & & \\
\hline Aesthetic outcomes & Good & $142(90.4 \%)$ \\
& Fair & $10(6.4 \%)$ \\
& Poor & $5(3.2 \%)$ \\
\hline
\end{tabular}

lacrimal glands which lie beneath the lateral part of the flap.

views with eyes open and $\mathbf{b}$ with eyes closed. $\mathbf{c}$ Twelvemonth postoperative views (combining epicanthoplasty) with eyes open and $\mathbf{d}$ with eyes closed.

In the current blepharoptosis correction surgeries for Asians, recurrence and poor double eyelid shape are still major complications faced by surgeons. The reason for these problems could be that traditional levator-based correction surgery is not the best solution for puffier Asian upper eyelids. These traditional levatorbased techniques only focus on enhancement of the levator muscle power, while neglecting the continuation of this power to the orbicularis 
Table 4 Results of complication outcomes $(N=225$ eyes)

\begin{tabular}{lll}
\hline No. of eyes (rate) & & \\
\hline $\begin{array}{l}\text { Complication } \\
\text { outcomes }\end{array}$ & Undercorrection & $12(5.3 \%)$ \\
& Overcorrection & $9(4 \%)$ \\
& $\begin{array}{l}\text { Residual } \\
\text { lagophthalmos }\end{array}$ & 0 \\
& Exposure keratitis & 0 \\
& Recurrence & $10(4.4 \%)$ \\
\hline
\end{tabular}

oculi muscle and skin. Moreover, fullness of the Asian upper eyelids can result in asymmetric scars when the eyes are closed and a deep-set crease when the eyes are opened (Fig. 5).

The upper eyelid can be divided into the anterior and posterior lamella; the levator aponeurosis acts as the main traction to lift the anterior lamella and Müller's muscle lifts the posterior lamella [12]. In our new technique, we enhanced the elevating power by advancing the levator aponeurosis and Müller's muscle as a whole flap, thus providing more reliable support and reducing the recurrence rate. Our

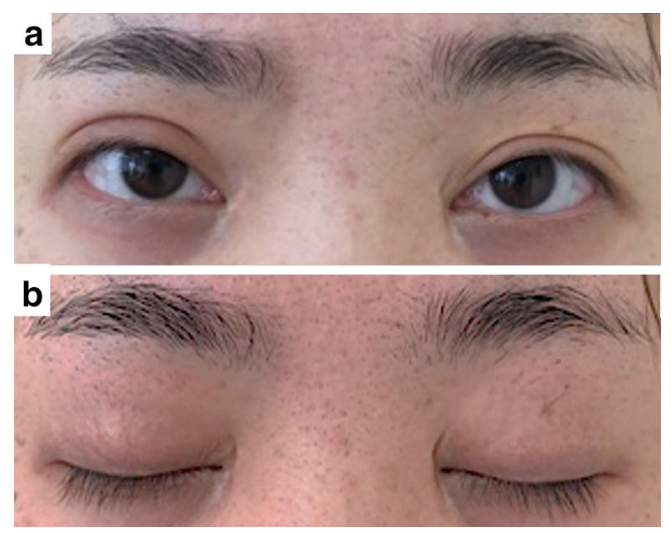

Fig. 5 Cases of unsatisfactory results after blepharoptosis surgery. a A female patient (27 years old) who had moderate blepharoptosis (right eye). She underwent blepharoptosis surgery 2 years ago elsewhere. Postoperative photos showed deep-set crease with eyes open and b asymmetric scars with eyes closed. c Another female patient ( 30 years old) who had bilateral blepharoptosis method also kept the orbicularis oculi muscles intact instead of being excised, as they are typically used in traditional surgical procedures to protect the function of eyelid closure [13]. The anchoring of the bridge on the tarsus provided a raw contact surface to create stable adhesion, which also contributed to restoring continuity between the anterior and posterior lamella.

Previously, the skin was sutured directly to the tarsus, often resulting in a sunken incision. To ensure that the postoperative incision was flat upon recovery, the skin was sutured over the free end of the bridge using our technique. Hence, the bridge terminated at the skin, thus achieving physiological correction of blepharoptosis.

In traditional levator resection or advancement surgeries, reconnection is achieved by scar tissue formation between the skin and aponeurosis. However, due to the cutting force of the sutures on the soft tissues, relapse and loosening are likely to occur. Moreover, our three-layer fixation, which includes firm tarsal fixation of the bridge, and our internal and external fixation procedure ensured the stability of the correction effect and minimised the recurrence rate (4.4\%), compared with the traditional levator procedure, with a recurrence rate of $10-15 \%$

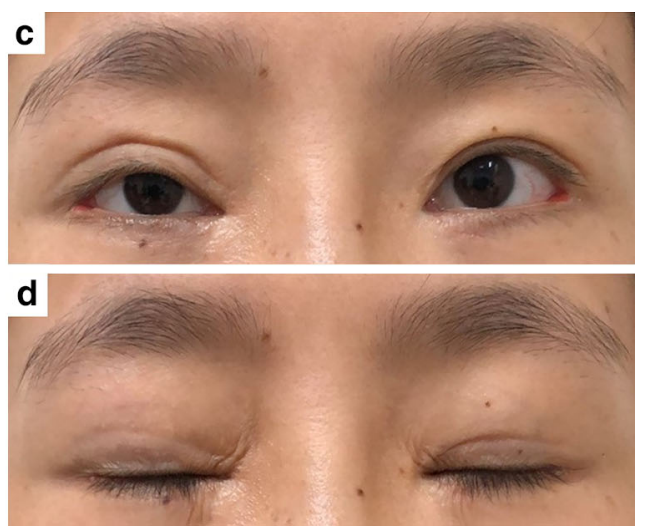

with right eye graded as moderate and left eye graded as mild. She underwent blepharoptosis surgery combined with lateral canthoplasty 3 years ago elsewhere. Postoperative photos showed deep-set and unnatural eyelid crease with eyes open and $\mathbf{d}$ asymmetric and depressed scars with eyes closed. The preoperative photos of these two patients were not accessible 
[14]. To avoid this situation, the scar tissue formation or sutures are replaced by bridge-orbicularis oculi muscle-dermis adhesion, as the reconnection procedure in our technique, which can theoretically prevent the formation of scar depression and has achieved optimal clinical results in our clinical practice.

Our study is an observational study that examined 157 patients with mild-to-moderate blepharoptosis to explore the efficacy of a new technique. We proposed a new concept, aesthetic correction of blepharoptosis among Asians, which strengthens the importance of aesthetics in addition to functional correction. However, our study included patients with a median age of 25.1 years (18-35 years), which indicates that our technique showed effectiveness in young Asians. Therefore, the efficacy of this technique remains to be explored in middle-aged and elderly patients. More high-quality and comparative studies with other blepharoptosis correction methods are necessary to explore the safety and efficacy of this technique.

\section{CONCLUSIONS}

Our new technique is effective in correcting mild-to-moderate blepharoptosis and at the same time can achieve optimal aesthetic outcomes. The long-term outcomes of this approach are being further explored.

\section{ACKNOWLEDGEMENTS}

Funding. This study was supported by the National Natural Science Foundation of China (nos. 81871576, 81701901, and 81801946). The journal's Rapid Service Fee was funded by Shanghai Municipal Key Clinical Specialty (shslczdzk00901).

Authorship. All the named authors meet the International Committee of Medical Journal Editors (ICMJE) criteria for authorship for this article, and take responsibility for the integrity of the work as a whole and have given their approval for this version to be published.
Author Contributions. Rui Jin and Jun Yang designed and supervised the study. Yirui Shen drafted all the versions of the manuscript. Wenjie Yu, Feixue Ding, Lin Lu, and Fei Liu advised on the successive drafts of the manuscript. Di Sun and Xusong Luo contributed to data acquisition and analysis.

Disclosures. Yirui Shen, Wenjie Yu, Feixue Ding, Lin Lu, Fei Liu, Di Sun, Xusong Luo, Rui Jin, and Jun Yang declare that they have no conflicts of interests. The authors declare no potential conflicts of interest with respect to the research, authorship, and publication of this article.

Compliance with Ethics Guidelines. We thank all the participants in this study. Informed consent was acquired from all the participants. All the procedures involving human subjects were conducted in accordance with the Declaration of Helsinki and have been approved by the Ethics Committee of Shanghai Ninth People's Hospital Affiliated to Shanghai Jiaotong University School of Medicine.

Data Availability. Data sharing is not applicable to this article since no datasets were generated or analysed during the current study.

Open Access. This article is licensed under a Creative Commons Attribution-NonCommercial 4.0 International License, which permits any non-commercial use, sharing, adaptation, distribution and reproduction in any medium or format, as long as you give appropriate credit to the original author(s) and the source, provide a link to the Creative Commons licence, and indicate if changes were made. The images or other third party material in this article are included in the article's Creative Commons licence, unless indicated otherwise in a credit line to the material. If material is not included in the article's Creative Commons licence and your intended use is not permitted by statutory regulation or exceeds the permitted use, you will need to obtain permission directly from the copyright holder. To view a copy of this licence, visit http://creativecommons.org/licenses/bync/4.0/. 


\section{REFERENCES}

1. Patel K, Carballo S, Thompson L. Ptosis. Dis Mon. 2017;63:74-9.

2. Fea A, Damato D, Actis AG, De Sanctis U, Actis G, Grignolo FM. Blepharoplastic: essential review. Minerva Chir. 2013;68:49-56.

3. Chang S, Lehrman C, Itani K, Rohrich R. A systematic review of comparison of upper eyelid involutional ptosis repair techniques: efficacy and complication rates. Plast Reconstr Surg. 2012;129: 149-57.

4. Ben Simon GJ, Lee S, Schwarcz RM, McCann JD, Goldberg RA. External levator advancement vs Muller's muscle-conjunctival resection for correction of upper eyelid involutional ptosis. Am J Ophthalmol. 2005;140:426-32.

5. Anderson RL, Dixon RS. Aponeurotic ptosis surgery. Arch Ophthalmol. 1979;97:1123-8.

6. Berlin AJ, Vestal KP. Levator aponeurosis surgery. A retrospective review. Ophthalmology. 1989;96: 1033-7.

7. McCulley TJ, Kersten RC, Kulwin DR, Feuer WJ. Outcome and influencing factors of external levator palpebrae superioris aponeurosis advancement for blepharoptosis. Ophthal Plast Reconstr Surg. 2003;19:388-93.
8. Lee JH, Nam SM, Kim YB. Blepharoptosis correction: levator aponeurosis-Müller muscle complex advancement with three partial incisions. Plast Reconstr Surg. 2015;135:388-95.

9. Jeong S, Lemke BN, Dortzbach RK, Park YG, Kang HK. The Asian upper eyelid: an anatomical study with comparison to the Caucasian eyelid. Arch Ophthalmol. 1999;117:907-12.

10. Harvey DJ, Iamphongsai S, Gosain AK. Unilateral congenital blepharoptosis repair by anterior levator advancement and resection: an educational review. Plast Reconstr Surg. 2010;126:1325-31.

11. Scoppettuolo E, Chadha V, Bunce C, Olver JM, Wright M. BOPSS. British Oculoplastic Surgery Society (BOPSS) national ptosis survey. Br J Ophthalmol. 2008;92:1134-8.

12. Kakizaki H, Zako M, Nakano T, Asamoto K, Miyaishi $\mathrm{O}$, Iwaki M. The levator aponeurosis consists of two layers that include smooth muscle. Ophthalmic Plast Reconstr Surg. 2005;21:281-4.

13. Hwang K. Surgical anatomy of the upper eyelid relating to upper blepharoplasty or blepharoptosis surgery. Anat Cell Biol. 2013;46:93-100.

14. Finsterer J. Ptosis: causes, presentation, and management. Aesthetic Plast Surg. 2003;27:193-204. 